\title{
Direct Model-Based Inversion for Improved Freehand Optical Ultrasound Imaging
}

\author{
Erwin J Alles*, ${ }^{*}$, Eleanor C Mackle*, ${ }^{*}$, Sacha Noimark ${ }^{*, \dagger}$, Adrien E Desjardins ${ }^{*, \dagger}$ \\ ${ }^{*}$ Wellcome / EPSRC Centre for Interventional and Surgical Sciences, University College London, London, UK \\ ${ }^{\dagger}$ Department of Medical Physics \& Biomedical Engineering, University College London, London, UK
}

\begin{abstract}
Optical ultrasound imaging uses light to both generate and detect pulse-echo ultrasound. Recently, we presented a fibre-optic optical ultrasound imaging probe comprising 64 sources and a single receiver that allowed for video-rate, freehand imaging. However, its low number of sources limited the image contrast when using Delay-and-Sum reconstruction. Here, we present an alternative image formation paradigm for optical ultrasound based on model-based inversion, where the low number of sources allows for direct (i.e., non-iterative) inversion under modest hardware requirements. The model accurately incorporates the aperture geometry, frequency-dependent source directivity, and performance variation across the aperture, thereby reducing image artefacts associated with these properties. The method achieves a $15 \mathrm{~dB}$ gain in image contrast compared to Delay-and-Sum, at a similar image formation time.

Index Terms-Model-based inversion, optical ultrasound, image formation, singular value decomposition, regularisation
\end{abstract}

\section{INTRODUCTION}

Biomedical ultrasound imaging is conventionally performed using imaging probes based on piezoelectric or capacitive micro-machined ultrasound transducers (cMUTs) [1]. While such probes generate high-quality images, their frequency and bandwidths (and thus penetration and resolution) are typically fixed during fabrication, and the presence of metal and electronics results in adverse interactions (e.g., interference or heating) with strong electromagnetic fields such as encountered during X-ray or magnetic resonance imaging.

Recently, an optical ultrasound (OpUS) imaging paradigm has been presented, which uses light to both generate and detect ultrasound. Ultrasound is generated via photoacoustic conversion of excitation light into ultrasound [2] at an optically absorbing coating deposited at the tip of an optical fibre. Back-scattered ultrasound signals are detected by optically resonant detectors, such as Fabry-Pérot cavities [3] or ring resonators [4], fabricated at the tip of another optical fibre. Such fibre-optic OpUS probes can have miniature lateral dimensions (diameter: $<1 \mathrm{~mm}$ ), exhibit immunity to electromagnetic interference, and emit highly broadband ultrasound (commonly a fractional bandwidth of $150-200 \%$ centered around $15 \mathrm{MHz}$ [5]) that allows for dynamic control over penetration depth and resolution [6].

High-quality fibre-optic OpUS imaging has been demonstrated using two types of imaging probes. First, two-fibre

This work was supported by the Wellcome Trust (203145Z/16/Z), the Engineering and Physical Sciences Research Council (EPSRC) (NS/A000050/1, EP/N021177/1, EP/S001506/1), and the Rosetrees Trust (PGS19-2/10006). probes (one transmitter, one receiver) have been mechanically scanned on the benchtop to generate $2 \mathrm{D}$ or $3 \mathrm{D}$ ex vivo images [3], [5], [7]-[12], or used in vivo to achieve Mmode imaging [13]. While these probes yielded high-quality images, acquisition times of minutes for $2 \mathrm{D}$, or hours for $3 \mathrm{D}$, prohibited clinical use. Second, a handheld imaging probe comprising a single fibre-optic ultrasound detector and 64 fibre-optic sources has been demonstrated to allow for video-rate, real-time $2 \mathrm{D}$ imaging using a flexible, handheld probe [14]. While this probe achieved a high frame rate, the applied image reconstruction algorithm combined with its low number of sources limited the image contrast.

Image reconstruction in OpUS typically relies on Delayand-Sum (DaS), which is equivalent to dynamic focussing [15]. This algorithm assumes that actual pulse-echo signal originating from an image pixel and detected across the aperture sums coherently, whereas other signal components (e.g. clutter, noise) average out to zero. While this works well for large numbers of channels (conventional electronic linear arrays comprise hundreds of elements each operating in duplex), this assumption is invalid for low channel counts such as observed in the handheld OpUS probe [14]. The image quality of low channel count OpUS images can be improved by exploiting cross-channel coherence using, for instance, shortlag spatial coherence (SLSC) [16] or Delay-Multiply-andSum (DMaS) [17]. However, such algorithms only yield small improvements for low channel counts, and in addition are nonlinear in nature which complicates image interpretation.

Here, we present an alternative image formation method based on model-based inversion, as commonly applied iteratively in photoacoustic imaging [18]. However, in this work we exploit the low channel count of the handheld OpUS imaging probe by performing direct instead of iterative model-based inversion. This direct model-based inversion (DMI) enables simultaneous inversion for a range of array properties (such as temporal source signature, source directivity, aperture geometry, and performance variation across the aperture) under only moderate computational hardware requirements and subsecond image formation time.

\section{Methods}

\section{A. Direct model-based inversion}

Model-based inversion in imaging is a class of techniques that assumes an accurate "forward model" is available to numerically predict the measured signals $\vec{B}$ for a given contrast $\vec{R}$. 


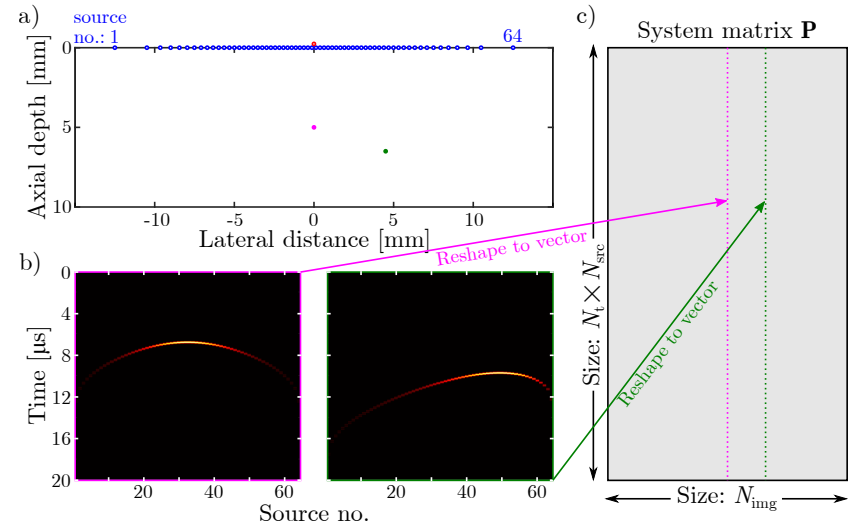

Fig. 1. Construction of system matrix P. a) Schematic of the image geometry, showing the source positions in blue, the detector position in red, and two point scatterers in magenta and green. b) Pulse-echo B-scans are computed for a point scatterer placed in each image pixel location using an acoustical model incorporating the probe geometry, temporal signature, source directivity, and performance variation across the aperture. For clarity, the A-scans were envelope-detected in this visualisation. c) These B-scans are reshaped into column vectors, and stored in the corresponding columns of the system matrix.

If this model is linear, the forward problem can be described as a matrix-vector multiplication

$$
\vec{B}=\mathbf{P} \vec{R},
$$

where $\mathbf{P}$ is a matrix describing the physical, spatial and temporal properties of the imaging system. In practice, however, the contrast function $\vec{R}$ is the variable of interest and only $\vec{B}$ is known.

In principle, equation 1 can be solved for $\vec{R}$ by computing the inverse of $\mathbf{P}$. However, this matrix is usually not invertible (for instance, this matrix is rarely square), and hence $\vec{R}$ can only be approximated by the matrix-vector multiplication

$$
\vec{R} \approx \tilde{\mathbf{P}}^{-1} \vec{B},
$$

where $\tilde{\mathbf{P}}^{-1}$ is the pseudo-inverse of system matrix $\mathbf{P}$. Similar to the forward problem, the contrast function (or image) can then be obtained via a single matrix-vector multiplication. Due to memory limitations, matrix $\tilde{\mathbf{P}}^{-1}$ can typically only be stored for modestly sized problems, such as those with low channel counts or small numbers of image pixels.

For the OpUS probe considered here, the construction of system matrix $\mathbf{P}$ is depicted in Fig. 1. A point scatterer of unit strength is placed in pixel $i$ in the image, which is equivalent to a binary contrast (reflectivity) function $\vec{R}$ where the corresponding element is set to 1 :

$$
\vec{R}_{j}=\left\{\begin{array}{ll}
1 & \text { if } j=i \\
0 & \text { otherwise }
\end{array},\right.
$$

where $j$ is the element counter. Next, an acoustic model is used to compute pulse-echo B-scan $B(k, t)$, where $k \in\left[1, N_{\text {src }}\right]$ and $t$ indicate the A-scan number and time, respectively. Note that only a single detector is used; consequently, the number of A-scans equals the number of sources $\left(N_{\mathrm{src}}\right)$. The resulting B- scan $B(k, t)$ is then reshaped into a column-vector to form the $i$-th column of system matrix $\mathbf{P}$. This process is repeated for all $N_{\text {img }}$ image pixels, resulting in a system matrix comprising $N_{\mathrm{t}} \times N_{\mathrm{src}} \times N_{\mathrm{img}}$ elements, where $N_{\mathrm{t}}$ is the number of time samples.

\section{B. Acoustic model}

Each of the 64 fibre-optic ultrasound sources was modelled as a circular piston transducer, and the pressure generated at the location of the point scatterer was computed using the spatio-temporal impulse response $H(r, t)$ implemented in the FOCUS toolbox for MATLAB [19] at a temporal sampling rate of $2.5 \mathrm{GHz}$. The piston transducers were modelled as velocity sources, and the generated pressure was hence given by the temporal convolution of the impulse response with the temporal derivative of the particle velocity at the transducer surface [20]. The single fibre-optic ultrasound detector exhibits a broadband and nearly omni-directional response [3], [21]; for simplicity it was therefore modelled as a point source using the free-space Green's function [1]. Finally, a band-pass filter $F(5-15 \mathrm{MHz})$ was applied to match the model to the experimental system, and the signals were down-sampled to a sampling rate of $62.5 \mathrm{MHz}$. To reduce memory consumption, all computations were performed in single precision.

For computational efficiency, the convolutions were performed in the frequency domain. For a single scatterer located in each image pixel $i$, the pulse-echo B-scan was computed as

$$
\begin{aligned}
B(k, t)=\mathcal{F}^{-1} & \left\{\hat{H}\left(\vec{r}_{\mathrm{img}, i}-\vec{r}_{\mathrm{src}, k}, \omega\right) .\right. \\
& \left.\frac{e^{-i \omega\left|\vec{r}_{\mathrm{img}, i}-\vec{r}_{\mathrm{rec}}\right| / c}}{4 \pi\left|\vec{r}_{\mathrm{img}, i}-\vec{r}_{\mathrm{rec}}\right|} \cdot i \omega \hat{v}(k, \omega) \cdot F(\omega)\right\},
\end{aligned}
$$

where $\vec{r}_{\mathrm{img}, i}, \vec{r}_{\mathrm{src}, k}$, and $\vec{r}_{\mathrm{rec}}$ are the coordinates of image pixel $i$, source $k$, and the single receiver, respectively, and $c$ is the speed of sound. Operators ${ }^{\wedge}$ and $\mathcal{F}^{-1}$ indicate spectral notation and the inverse Fourier transform, respectively.

The particle velocity at the transducer surface $v(k, t)$ was computed for each of the sources via stabilised spectral division (or Wiener filtering) of experimental pulse-echo data (obtained for a single, stationary point scatterer) by the impulse response, i.e.,

$$
\hat{v}(k, \omega)=\frac{\hat{B}(k, \omega)(i \omega \hat{H})^{*}}{i \omega \hat{H}(i \omega \hat{H})^{*}+[\epsilon \cdot \max |i \omega \hat{H}|]^{2}},
$$

where $\hat{H} \equiv \hat{H}\left(\vec{r}_{\mathrm{scat}}-\vec{r}_{\mathrm{src}, k}, \omega\right)$ was introduced for brevity, $\vec{r}_{\text {scat }}$ is the location of the physical point scatter, and stabilisation constant $\epsilon=1 \%$ was determined empirically to avoid over-fitting of noise.

\section{Numerical implementation and computational load}

Pseudo-inversion of the system matrix $\mathbf{P}$ was performed via singular value decomposition (SVD), resulting in an approxi- 

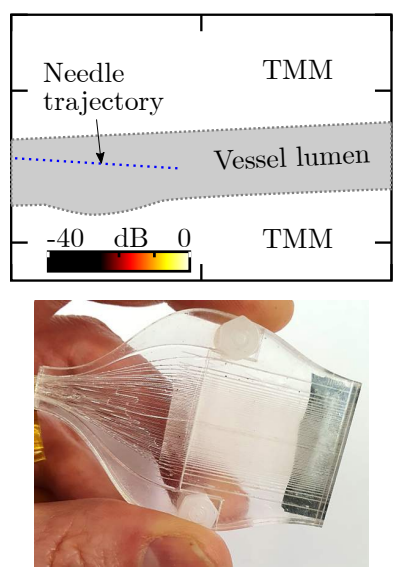
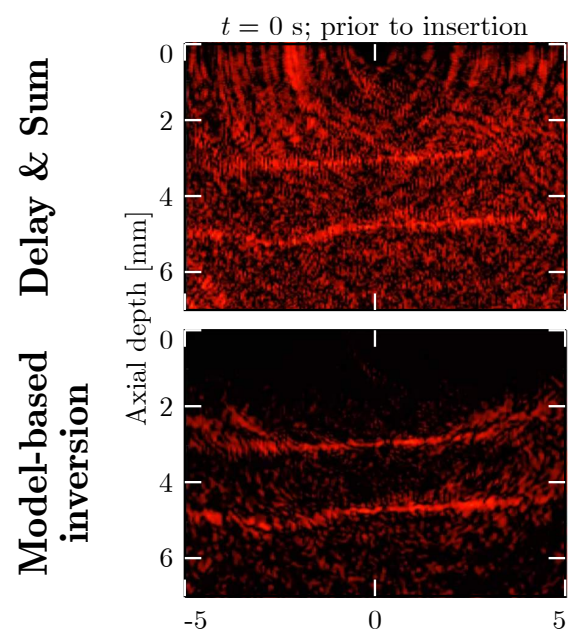

$t=6 \mathrm{~s}$; during insertion

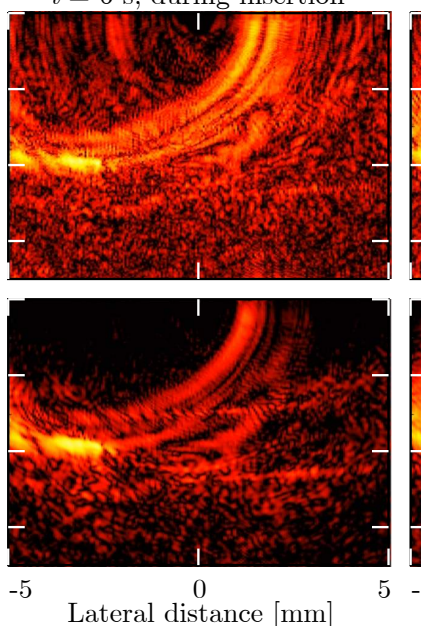

$t=7 \mathrm{~s} ;$ fully inserted
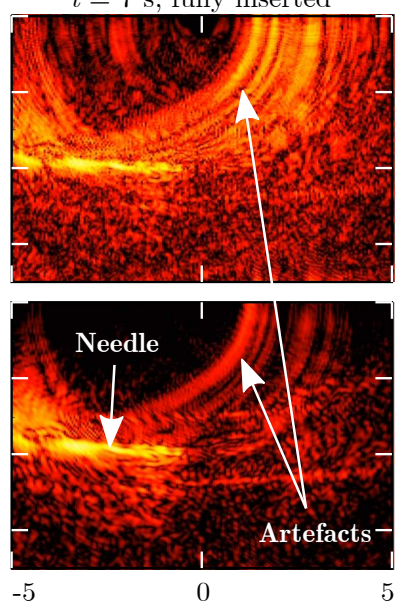

Fig. 2. Direct model-based inversion (DMI) versus conventional Delay-and-Sum reconstruction. Left - Schematic of the phantom geometry, vessel lumen and needle trajectory (top) and photograph of the optical ultrasound imaging probe (bottom). Right - Optical ultrasound images at different time points acquired using Delay-and-Sum (top) and DMI image formation (bottom) under Tikhonov regularisation ( $\alpha=1 \%$ ). All frames are shown on the same logarithmic scale using a $40 \mathrm{~dB}$ dynamic range. TMM: tissue-mimicking material.

mate decomposition (in the least-squares sense)

$$
\mathbf{P} \approx \mathbf{U S V}^{\prime}
$$

where $\mathbf{U}$ and $\mathbf{V}$ are unitary matrices, operator ' indicates the transpose, and $\mathbf{S}$ is a diagonal matrix containing the singular values. The singular value decomposition was truncated to obtain a square matrix $\mathbf{S}$ (equivalent to using the 'econ' flag in MATLAB). Pseudo-inversion was then achieved by

$$
\tilde{\mathbf{P}}^{-1} \approx \mathbf{V S}^{-1} \mathbf{U}^{\prime}
$$

where $\mathbf{S}^{-1}$ is computed using two regularisation techniques to avoid over-fitting of noise: Moore-Penrose, where

$$
\mathbf{S}_{i i}^{-1}=\left\{\begin{array}{ll}
1 / \mathbf{S}_{i i} & \text { if } \mathbf{S}_{i i} \geq \alpha \cdot \max \mathbf{S} \\
0 & \text { otherwise }
\end{array},\right.
$$

with stabilisation parameter $0 \leq \alpha \leq 1$, and Tikhonov (or $\mathrm{L}_{2}$ ) regularisation, where

$$
\mathbf{S}_{i i}^{-1}=\frac{\mathbf{S}_{i i}}{\mathbf{S}_{i i}^{2}+(\alpha \cdot \max \mathbf{S})^{2}} .
$$

In this work, the image measured $10 \mathrm{~mm}$ by $7 \mathrm{~mm}$, with pixel dimensions of $50 \mu \mathrm{m}$ (lateral) by $25 \mu \mathrm{m}$ (axial), resulting in $N_{\text {img }}=56481$ pixels. Temporally, $N_{\mathrm{t}}=1004$ samples were simulated or recorded, at a sampling rate of $62.5 \mathrm{MHz}$, for all $N_{\mathrm{src}}=64$ channels. System matrix $\mathbf{P}$ thus required $13.5 \mathrm{~GB}$ of memory to store in single precision, and was computed in approximately 37 minutes. During SVD, the matrices $\mathbf{U}, \mathbf{S}$ and $\mathbf{V}$ combined required an additional $37.3 \mathrm{~GB}$ of memory. Pseudo-inverse $\tilde{\mathbf{P}}^{-1}$ was computed inplace to overwrite $\mathbf{P}$ (thus avoiding $13.5 \mathrm{~GB}$ of memory). Once the SVD and pseudo-inverse were computed (which required 11.4 hours), all other matrices were cleared, and DMI image formation required just $13.5 \mathrm{~GB}$ of memory and $0.6 \mathrm{~s}$ per frame on an $17-9700 \mathrm{~K}$ CPU. By comparison, a single DaS frame was reconstructed in $0.51 \mathrm{~s}$ on the same hardware.

\section{Experimental design}

To demonstrate DMI experimentally, a tissue-mimicking phantom comprising a wall-less blood vessel was fabricated in $10 \%$ w/w poly(vinyl) alcohol (PVA) cryogel [22] and submerged in water. A needle (23G) was inserted into the vessel lumen (see Fig. 2) under continuous 2D OpUS imaging at a frame rate of $11 \mathrm{~Hz}$. Image frames were reconstructed using conventional DaS and DMI, under both Moore-Penrose and Tikhonov regularisation for various values of $\alpha$.

\section{RESULTS}

DMI under Tikhonov regularisation $(\alpha=1 \%)$ achieved a significant improvement in image quality compared to conventional $\mathrm{DaS}$ reconstruction (Fig. 2). Image artefacts were strongly reduced, while actual signals from the vessel wall and needle were retained. This resulted in a $15 \mathrm{~dB}$ gain in image contrast. Tikhonov regularisation utilised all singular values when computing the pseudo-inverse (after stabilisation; see Eq. 9), whereas Moore-Penrose regularisation disregarded a large proportion of the singular values (Eq. 8). Consequently, Tikhonov regularisation yielded more accurate images, regardless of stabilisation parameter $\alpha$ (Fig. 3). For Tikhonov regularisation, a value of $\alpha=1 \%$ was empirically determined to yield the best DMI images. Moore-Penrose regularisation using $\alpha=10 \%$ retained too few singular values to form a meaningful image.

\section{Discussion AND CONCLUSION}

This paper shows how direct model-based inversion (DMI) image formation can lead to a significant reduction in image artefacts and clutter compared to conventional DaS reconstruction, resulting in a $15 \mathrm{~dB}$ gain in contrast. The underlying model includes a range of factors, such as array geometry, 


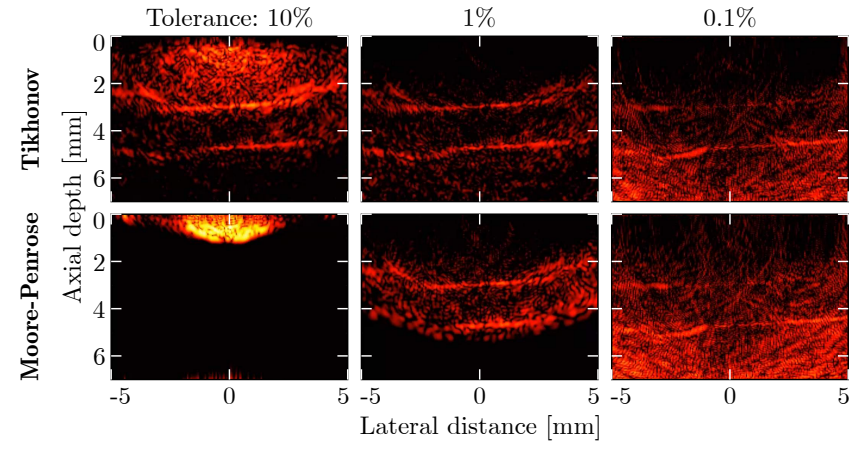

Fig. 3. The effect of regularisation. Direct model-based inversion images (at $t=0 \mathrm{~s}$ ) obtained using Tikhonov (top) and Moore-Penrose (bottom) regularisation, for three values of stabilisation parameter $\alpha=[10 \%, 1 \%, 0.1 \%]$.

number and location of transducer elements, source signature and directivity, and performance variation across the aperture, which are simultaneously accounted for upon inversion.

However, the contrast gain was limited by saturation of the A-scans obtained experimentally due to the high echogenicity of the needle, which was not accounted for in the model. Further improvements might be possible if this saturation can be avoided. In addition, other regularisation techniques (for instance, total variation or generalised Tikhonov) might yield even better images. Looking at Fig. 3, the optimal value of the regularisation parameter $\alpha$ appears to vary with axial depth; thus, a depth-dependent value for $\alpha$ could be considered.

The presented DMI approach only considers 2D imaging and in-plane events. This means that out-of-plane clutter is not accurately accounted for. While in principle this could be included in the model, the computational requirements render a direct inversion unlikely in this case. In addition, a homogeneous speed of sound is assumed, and attenuation is neglected as incorporating and inverting for these effects would only be possible using iterative methods, which are unrealistic for real-time applications. Despite these limitations, however, the significant improvement in image quality suggests the numerical model for the imaging system is highly accurate. The image formation accuracy could be further improved using a fully experimentally-derived system matrix that incorporates the electronics, optics, and data acquisition systems.

The method requires modest computational capabilities that are available in modern workstations, and requires image formation times that are similar to those of conventional DaS. The singular value decomposition, pseudo-inversion, and matrix-vector multiplications could each be significantly accelerated using modern Graphical Processing Unit (GPU) cards (currently equipped with up to 48 GB of VRAM memory), which could result in image formation times in the order of tens of milliseconds. Thus, DMI image formation shows great promise for improving the image quality of OpUS in real-time, and could be applied to other ultrasound modalities featuring low channel counts, such as intravascular ultrasound (IVUS) or systems with sparse arrays.

\section{REFERENCES}

[1] Richard S C Cobbold. Foundations of Biomedical Ultrasound. Oxford University Press, USA, New York, 2007.

[2] Paul C Beard. Biomedical photoacoustic imaging. Interface Focus, 1(4):602-631, 2011

[3] James A Guggenheim, Jing Li, Thomas J Allen, et al. Ultrasensitive plano-concave optical microresonators for ultrasound sensing. Nature Photonics, 11(11):714-719, 2017.

[4] Wouter J Westerveld, Md Mahmud-Ul-Hasan, Rami Shnaiderman, et al Sensitive, small, broadband and scalable optomechanical ultrasound sensor in silicon photonics. Nature Photonics, pages 1-5, 2021.

[5] Sacha Noimark, Richard J Colchester, Ben J. Blackburn, et al. Carbonnanotube-PDMS composite coatings on optical fibres for all-optical ultrasound imaging. Advanced Functional Materials, 26(35), 2016.

[6] Erwin J Alles, Richard J Colchester, and Adrien E Desjardins. Adaptive all-optical ultrasound imaging through temporal modulation of excitation light. In Proceedings IEEE IUS, pages 1-9. IEEE, 2018.

[7] E Biagi, S Cerbai, L Masotti, et al. Fiber optic broadband ultrasonic probe for virtual biopsy: Technological solutions. Journal of Sensors, 2010, 2010.

[8] Bao-Yu Hsieh, Sung-Liang Chen, Tao Ling, L Jay Guo, and Pai-Chi Li. All-optical scanhead for ultrasound and photoacoustic imaging - imaging mode switching by dichroic filtering. Photoacoustics, 2(1):39-46, 2014.

[9] Richard J Colchester, Edward Z Zhang, Charles A Mosse, Paul C Beard, Ioannis Papakonstantinou, and Adrien E Desjardins. Broadband miniature optical ultrasound probe for high resolution vascular tissue imaging. Biomedical Optics Express, 6(4):1502-1511, 2015.

[10] Sacha Noimark, Richard J Colchester, Radhika K Poduval, et al Polydimethylsiloxane composites for optical ultrasound generation and multimodality imaging. Advanced Functional Materials, 28(9), 2018.

[11] Richard J Colchester, Callum Little, George Dwyer, et al. All-optical rotational scan ultrasound imaging. Scientific Reports, 2019.

[12] Yinlong Zhang, Yizhi Liang, Long Jin, and Baiou Guan. $125 \mu \mathrm{m}$ fiber based all-optical ultrasound probes for pulse-echo imaging. Chinese Optics Letters, 17(7):070604, 2019.

[13] Malcolm C Finlay, Charles A Mosse, Richard J Colchester, et al. Through-needle all-optical ultrasound imaging in vivo: a preclinical swine study. Light: Science \& Applications, 6(12):e17103, 2017.

[14] Erwin J Alles, Eleanor C Mackle, S Noimark, Edward Z Zhang, Paul C Beard, and Adrien E Desjardins. Freehand and video-rate all-optical ultrasound imaging. Ultrasonics, 116:106514, 2021.

[15] Jørgen Arendt Jensen, Svetoslav Ivanov Nikolov, Kim Løkke Gammelmark, and Morten Høgholm Pedersen. Synthetic aperture ultrasound imaging. Ultrasonics, 44:e5-e15, 2006.

[16] Muyinatu A Lediju, Gregg E Trahey, Brett C Byram, and Jeremy J Dahl. Short-lag spatial coherence of backscattered echoes: Imaging characteristics. IEEE transactions on ultrasonics, ferroelectrics, and frequency control, 58(7):1377-1388, 2011.

[17] Giulia Matrone, Alessandro Stuart Savoia, Giosuè Caliano, and Giovanni Magenes. The delay multiply and sum beamforming algorithm in ultrasound B-mode medical imaging. IEEE transactions on medical imaging, 34(4):940-949, 2015.

[18] Benjamin T Cox, Jan G Laufer, Paul C Beard, and Simon R Arridge. Quantitative spectroscopic photoacoustic imaging: a review. Journal of biomedical optics, 17(6):061202, 2012.

[19] Robert J. McGough. FOCUS: Fast Object-Oriented C++ Ultrasound Simulator, www.egr.msu.edu/ fultras-web/index.php. Accessed: 24 May 2020.

[20] JC Lockwood and JG Willette. High-speed method for computing the exact solution for the pressure variations in the nearfield of a baffled piston. The Journal of the Acoustical Society of America, 53(3):735741, 1973.

[21] Edward Z Zhang and Paul C Beard. Characteristics of optimized fibreoptic ultrasound receivers for minimally invasive photoacoustic detection. In Proceedings SPIE BiOS, pages 932311-932311-9. International Society for Optics and Photonics, 2015.

[22] Eleanor C Mackle, Efthymios Maneas, Callum Little, et al. Wall-less vascular poly (vinyl) alcohol gel ultrasound imaging phantoms using 3D printed vessels. In Design and Quality for Biomedical Technologies XII, volume 10870, page 108700P. SPIE, 2019. 